\title{
Carbon Nanotubes/Gold Nanoparticles Composite Film for the Construction of a Novel Amperometric Choline Biosensor
}

\author{
Baoyan Wu, ${ }^{1}$ Zhongmin Ou, ${ }^{1}$ Xiaojuan Ju, ${ }^{2}$ and Shihua $\mathrm{Hou}^{3}$ \\ ${ }^{1}$ MOE Key Laboratory of Laser Life Science \& Institute of Laser Life Science, College of Biophotonics, \\ South China Normal University, Guangzhou 510631, China \\ ${ }^{2}$ University Hospital, South China Normal University, Guangzhou 510631, China \\ ${ }^{3}$ School of Electronic and Information Engineering, South China University of Technology, Guangzhou 510641, China \\ Correspondence should be addressed to Shihua Hou, shhou@scut.edu.cn
}

Received 17 July 2011; Revised 26 September 2011; Accepted 3 October 2011

Academic Editor: Yanqiu Zhu

Copyright () 2011 Baoyan Wu et al. This is an open access article distributed under the Creative Commons Attribution License, which permits unrestricted use, distribution, and reproduction in any medium, provided the original work is properly cited.

This study develops a facile method to fabricate a novel choline biosensor based on multiwalled carbon nanotubes (MWCNTs) and gold nanoparticles (AuNPs). Chitosan, a natural biocompatible polymer, was used to solubilize MWCNTs for constructing the aqueous Chit-MWCNTs solution. Then Chit-MWCNTs were first dropped on the surface of a cleaned platinum electrode. Finally, a thiolated silica sol containing AuNPs and choline oxidase (ChOx) was immobilized on the surface of the Chit-MWCNTsmodified electrode. The MWCNTs/AuNPs/Pt electrode showed excellent electrocatalytic activity for choline. The resulting choline biosensor showed high sensitivity of choline $(3.56 \mu \mathrm{A} / \mathrm{mM})$, and wide linear range from 0.05 to $0.8 \mathrm{mM}$ with the detection limit of $15 \mu \mathrm{M}$. In addition, good reproducibility and stability were obtained.

\section{Introduction}

Choline, a precursor of acetylcholine and phosphatidylcholine, is an essential nutrient required by the body for healthy cell membrane function [1-3]. Without choline, many fat-based nutrients and waste products could not pass in and out of our cells [4]. There is undisputed need for the measurement of neurochemical substances [5]. However, the measurement of choline is difficult because the concentrations of choline in the samples are quite low [6].

As the most promising alternative technique of choline determination, enzyme-based biosensors have attracted wide attention because they can offer simple and reliable means for the analysis $[4,6]$. For example, Bai et al. provided a sensitive and anti-interference choline biosensor based on a biocomposite composed of chitosan hydrogel, choline oxidase (ChOx), and $\mathrm{MnO}_{2}$ nanoparticles [4]. Another example is that presented by Song et al. [7]. They provided a choline biosensor based on the immobilization of $\mathrm{ChOx}$ into a sol-gel silicate film on the multiwall carbon nanotubes (MWCNTs) modified platinum electrode, exhibiting good sensitivity and stability. Recently, Frey et al. developed a novel functionalisation method for the deposition of ChOx and anti-interference layers on the microelectrodes, providing the evidence of adequate biosensor performances for the detection of choline in the physiologically relevant concentration ranges [5].

The physical and chemical properties of the materials used in the construction of biosensors have got significant influence on their performance [8]. The application of carbon nanotubes in enzyme-based biosensors has attracted the attention of many researchers due to their unique physical and chemical properties, which provided high surface areas for enzyme loading and a compatible microenvironment, and its ability to promote the electron transfer between the biomolecules and electrode surface has been extensively studied [9-11]. Meanwhile, gold nanoparticles (AuNPs) are one of the most intensively studied and applied metal nanoparticles in amperometric biosensors owing to its stable physical and chemical properties, catalytic activity, and small dimensional size [12-14]. Therefore, the composite composed of carbon nanotubes and AuNPs, an area of intense research, has been widely used for the fabrication of biosensors. It has been demonstrated that the carbon nanotubes 
and AuNPs composite can not only conserve or enhance the intrinsic properties of the particles, but also offer an effective approach to enhance the properties of biosensors [10, 1517]. For example, our group developed an amperometric glucose biosensor based on self-assembly of multilayer films composed of MWCNTs, AuNPs, and glucose oxidase for the specific detection of glucose, providing a promising alternative mode to design amperometric biosensors [10]. $\mathrm{Hu}$ et al. also demonstrated that MWCNTs/AuNPs composite is particularly promising for novel, highly efficient photoelectrochemical cells and sensor devices [16]. More recently, Zhang et al. introduced the deposition of AuNPs on a MWCNT-based composite film immobilized on an electrode interface and found that the modified electrode exhibited a variety of good electrochemical characteristics including a relatively high sensitivity and long-term stability [17].

Taking account of the advantages of MWCNTs and AuNPs, the main focus of the present study was to construct a novel choline biosensor based on MWCNTs and AuNPs composite film. To meet the desired requirements, ChitMWCNTs were first dropped on the surface of a Pt electrode, and then a thiolated silica sol containing AuNPs and ChOx was immobilized on the surface of the Chit-MWCNTs modified electrode to construct a MWCNTs/AuNPs/Ptbased amperometric choline biosensor. The electrocatalytic behaviors of the resulting choline biosensor are investigated and discussed.

\section{Experimental}

2.1. Reagents. Choline oxidase (EC 1.1.3.17, 17 units/mg protein, from alcaligenes species), (3-mercaptopropyl) trimethoxy silane (MPTMOS) and chitosan (low molecular weight, 75-85\% deacetylated) were purchased from SigmaAldrich. MWCNTs (>50 nm diameter, length $0.5-1 \mu \mathrm{m}$, and $>95 \%$ purity) were obtained from Chengdu Institute of Organic Chemistry, Chinese Academy of Science (China). Chloroauric acid hydrated $\left(\mathrm{HAuCl}_{4} \cdot 4 \mathrm{H}_{2} \mathrm{O}\right)$ were purchased from Sinopharm Chemical Reagent Co. (China). All the reagents were of analytical grade and used without further purification. The high-purity deionized water (resistance $>18 \mathrm{M} \Omega \cdot \mathrm{cm}$ ) was used throughout this work. Phosphatebuffered saline (PBS, pH 8.0) buffer was employed as supporting electrolyte. All experiments were performed in $\mathrm{PBS}$ at room temperature, approximately $25^{\circ} \mathrm{C}$.

\subsection{Preparation of the Biosensor}

2.2.1. Preparation of Chit-MWCNTs. MWCNTs $(1 \mathrm{~g} / \mathrm{L})$ were sonicated in chitosan solution $(2 \mathrm{~g} / \mathrm{L}$, in acetate buffer, $\mathrm{pH}$ 5.0 ), and centrifuged at 10,000 rpm for $10 \mathrm{~min}$. The sediment comprising of impurities aggregates and bundles of nanotubes at the bottom of the centrifuge tube was discarded, and the supernatant containing Chit-MWCNTs was collected and filtrated through a $100 \mathrm{KDa}$ filter (Millipore) to remove excess chitosan.

2.2.2. Preparation of AuNPs. All glassware used in the following procedure was cleaned in a bath of freshly prepared solution $\left(3: 1 \mathrm{HNO}_{3}-\mathrm{HCl}\right)$, thoroughly washed with water, and dried prior to use. The AuNPs were prepared according to the literature [18] by adding sodium citrate to a boiling $\mathrm{HAuCl}_{4}$ aqueous solution. The solution was kept boiling for 15 min under vigorous stirring, then allowed to cool down to room temperature, and stored at $4^{\circ} \mathrm{C}$.

2.2.3. Preparation of Silica Sol. Silica sol was prepared according to the literatures $[19,20]$. Briefly, MPTMOS with water at a $1: 4$ ratio, $10 \%$ of ethanol, and $3.3 \%$ of $0.1 \mathrm{M}$ hydrochloric acid were mixed and sonicated for $30 \mathrm{~min}$ until a clear and homogenous solution resulted and was subsequently stored at room temperature for 2-3 h.

2.2.4. Configuration of Choline Biosensor. Prior to the experiment, the bare Pt electrode was polished with chamois leather containing $0.05 \mu \mathrm{m}$ alumina slurry, rinsed thoroughly with water, sonicated in water and absolute ethanol, and dried at room temperature. Then, $5 \mu \mathrm{L}$ of Chit-MWCNTs was dropped on the surface of a cleaned Pt electrode and allowed to dry at room temperature. A casting solution was prepared by mixing sol-gel solution with $\mathrm{ChOx}$ and AuNPs at a 1:2:2 volume ratio. After carefully mixing, $10 \mu \mathrm{L}$ of the freshly prepared casting solution was dropped immediately on the surface of bare and Chit-MWCNTs modified Pt electrodes, regarded as AuNPs/Pt electrode and MWCNTs/AuNPs/Pt electrode, respectively, and allowed to dry at $4^{\circ} \mathrm{C}$.

2.3. Scanning Electron Microscope (SEM) Measurements. Surface image of the substrate modified by Chit-MWCNTs was obtained by SEM (QUANTA 200, FEI Co.) measurement operated at an accelerating voltage of $25.0 \mathrm{kV}$.

2.4. Transmission Electron Microscope (TEM) Measurements. To examine the size of AuNPs, the sample was observed by a JEM-2100HR TEM by dropping $5 \mu \mathrm{L}$ of the solution on the top of a TEM grid.

2.5. Optical Spectra Measurements. The absorption spectrum of AuNPs was obtained using a UV/VIS spectrometer (Lambda 35, Perkin-Elmer, USA).

2.6. Electrochemical Measurements. Electrochemical measurements were carried out by a Potentiostat-Galvanostat (EG \& G PARC Model 283 with a software M270). All electrochemical experiments were carried out using a conventional three-electrode system comprising the AuNPs and MWCNTs modified Pt electrode as the working electrode, a $\mathrm{Pt}$ wire as the auxiliary electrode, and $\mathrm{Ag} / \mathrm{AgCl}$ (saturated $\mathrm{KCl})$ as the reference electrode in an electrochemical cell filled with $20 \mathrm{~mL}$ of PBS at room temperature. In steady-state amperometric experiment, the potential was set at $+600 \mathrm{mV}$ under gently magnetic stirring. 


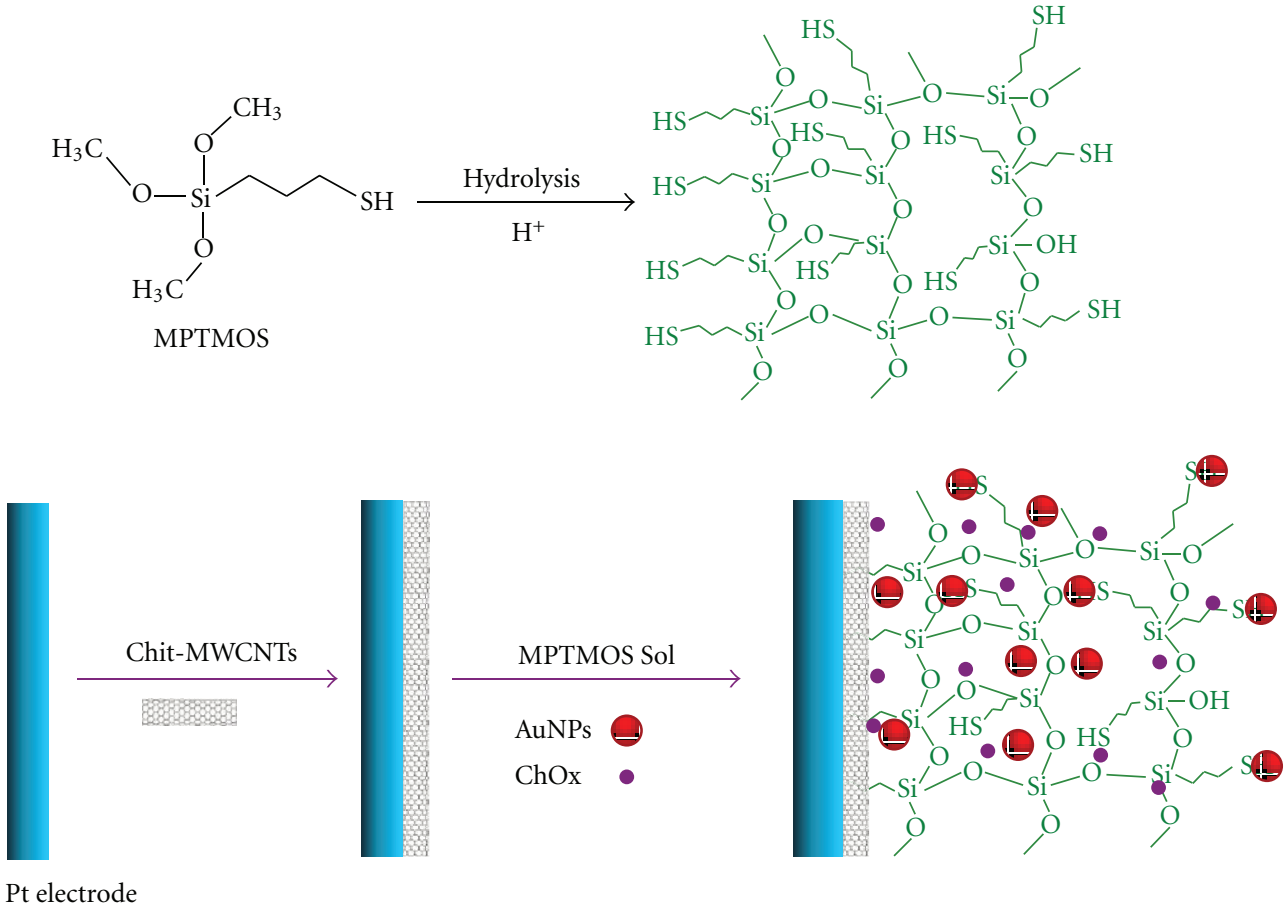

Figure 1: Scheme of preparation of the ChOx amperometric biosensor for choline based on AuNPs, MWCNTs, and silica sol-gel of MPTMOS.

\section{Results and Discussion}

Biomedical application potentials of carbon nanotubesbased materials have been investigated intensively in recent years [21]. In this work, a novel amperometric choline biosensor based on MWCNTs, AuNPs, and silica sol-gel of MPTMOS has been developed, as shown in Figure 1. Firstly, MWCNTs were sonicated in chitosan solution and dropped on the surface of a cleaned Pt electrode. Then, the sol-gel solution composed of AuNPs and ChOx was dropped on the surface of the Chit-MWCNTs modified Pt electrode. The working mechanism of the resulting choline biosensor for choline is based on the following sequential biochemical reactions:

$$
\text { Choline }+\mathrm{O}_{2} \stackrel{\text { ChOx }}{\longrightarrow} \text { betaine aldehyde }+\mathrm{H}_{2} \mathrm{O}_{2}
$$

betaine aldehyde $+\mathrm{O}_{2}+\mathrm{H}_{2} \mathrm{O} \stackrel{\mathrm{ChOx}}{\longrightarrow}$ betaine $+\mathrm{H}_{2} \mathrm{O}_{2}$

In the presence of oxygen, $\mathrm{ChOx}$ converts choline into betaine and hydrogen peroxide $\left(\mathrm{H}_{2} \mathrm{O}_{2}\right)$. Hydrogen peroxide, an electrochemically active product, is easily oxidized on electrode. The oxidation current of hydrogen peroxide can be used as a measure of choline.

The microscopic morphology of Chit-MWCNTs prepared by strong stirring and ultrasonication was shown in Figure 2(a), which can be stable for at least 1 month at room temperature without visible flocculation, evidenced by the homogeneous black color of Chit-MWCNTs solution (Figure 2(d)). The diameter of AuNPs was about $15 \mathrm{~nm}$, as shown in Figure 2(b), which was confirmed by the UVvis absorption spectrum (Figure 2(c)). AuNPs presented an absorption peak at $520 \mathrm{~nm}$ in the range from 400 to $600 \mathrm{~nm}$, which is the characteristic absorbance of AuNPs.

MPTMOS is a bifunctional molecule that contains both trimethoxysilane and thiol functional groups. Trimethoxysilane groups can undergo hydrolysis and condensation reaction to form a covalently linked siloxane network which is used for physical encapsulation of ChOx and AuNPs. Meanwhile, the thiol groups can be used for the covalent attachment of AuNPs to sol-gel network. It has been demonstrated that AuNPs with the diameter of $20 \mathrm{~nm}$ can be both diffused into and on the surface of the sol-gel network [22] and can generate a continuous array of AuNPs throughout the sol-gel network [23]. In view of the fact that the diameter of AuNPs used in this work was about $15 \mathrm{~nm}$, AuNPs can be immobilized both inside and on the surface of the sol-gel, providing a necessary conduction pathway between the immobilized $\mathrm{ChOx}$ and the surface of the $\mathrm{Pt}$ substrate. Therefore, the resulting choline biosensor will exhibit the benefits of sol-gel, nanoparticles, and enzymebased amperometric biosensor.

Figure 3 shows cyclic voltammograms of AuNPs (a and c), MWCNTs/AuNPs (b and d) modified Pt electrode in PBS containing no choline ( $\mathrm{a}$ and $\mathrm{b}$ ), and $1.6 \mathrm{mM}$ choline ( $\mathrm{c}$ and d) in the applied potential range from 0 to $600 \mathrm{mV}$ with a scan rate of $50 \mathrm{mV} / \mathrm{s}$, respectively. The response of AuNPs/Pt electrode and MWCNTs/AuNPs/Pt electrode in PBS displayed a low background current. Upon addition of $1.6 \mathrm{mM}$ choline in PBS, a large oxidation current was observed, confirming that $\mathrm{ChOx}$ catalyzed the oxidation reaction of choline and the resultant $\mathrm{H}_{2} \mathrm{O}_{2}$ was electrooxidized at the electrodes. The current of MWCNTs/AuNPs/Pt electrode was 


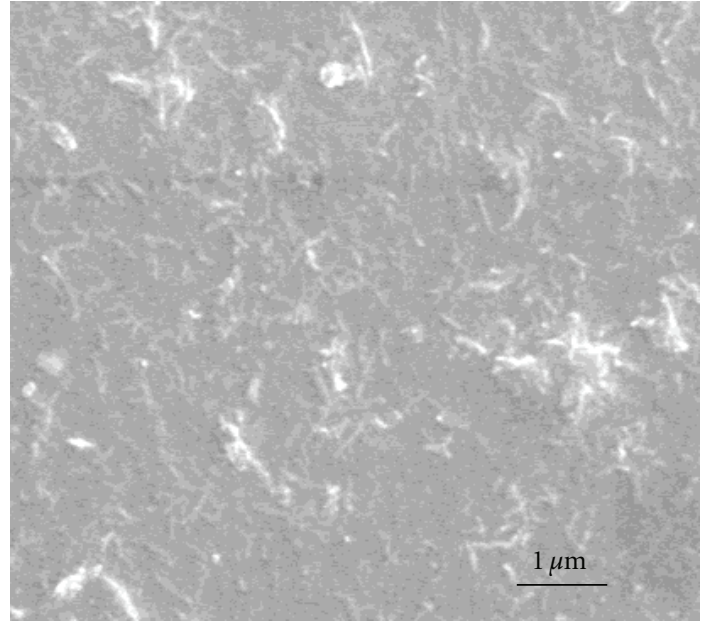

(a)

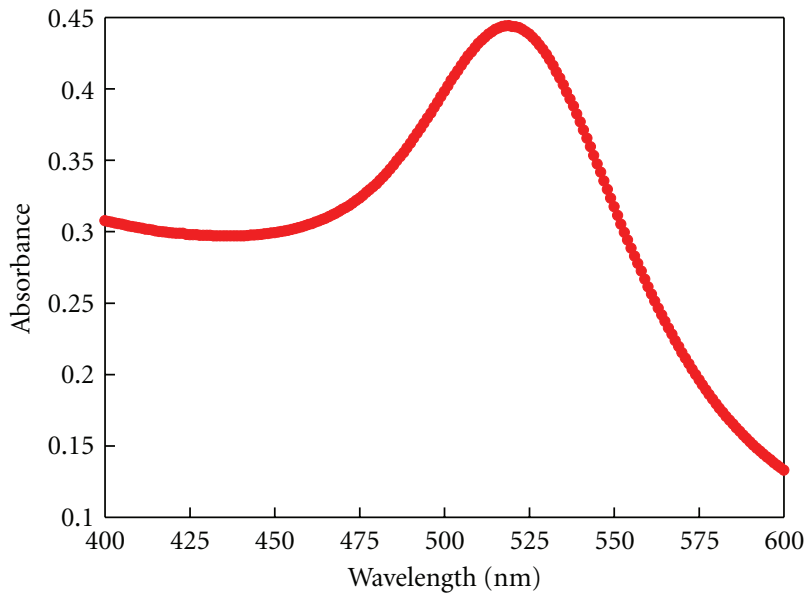

(c)

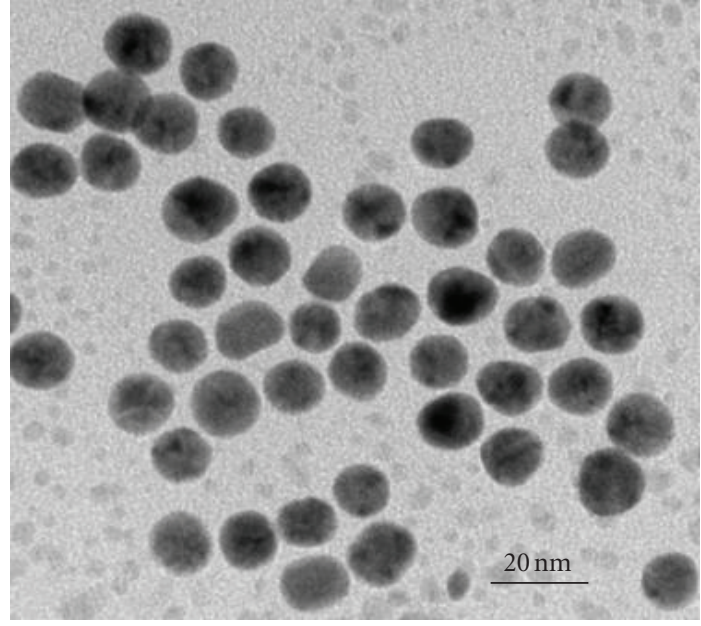

(b)

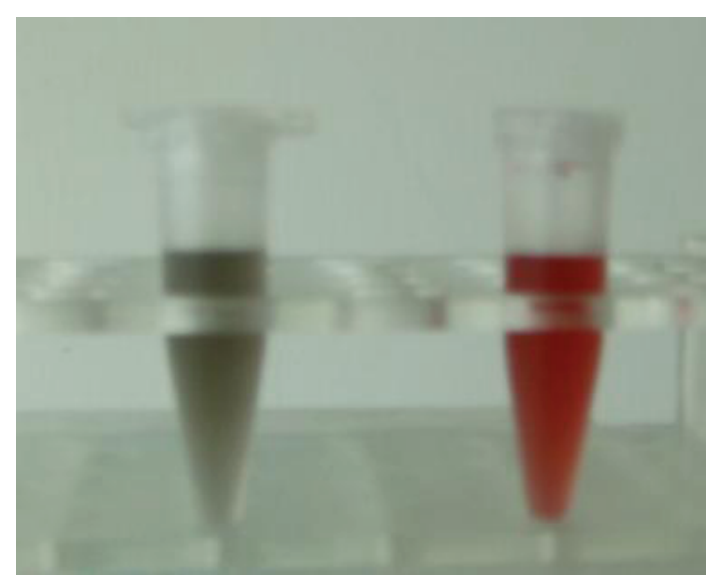

(d)

FIgURE 2: SEM image of Chit-MWCNTs (a), TEM image and UV-visible absorbance spectrum of AuNPs with an average diameter of about $15 \mathrm{~nm}$ (b and c), and a photo of Chit-MWCNTs solution (d, left) and AuNPs (d, right).

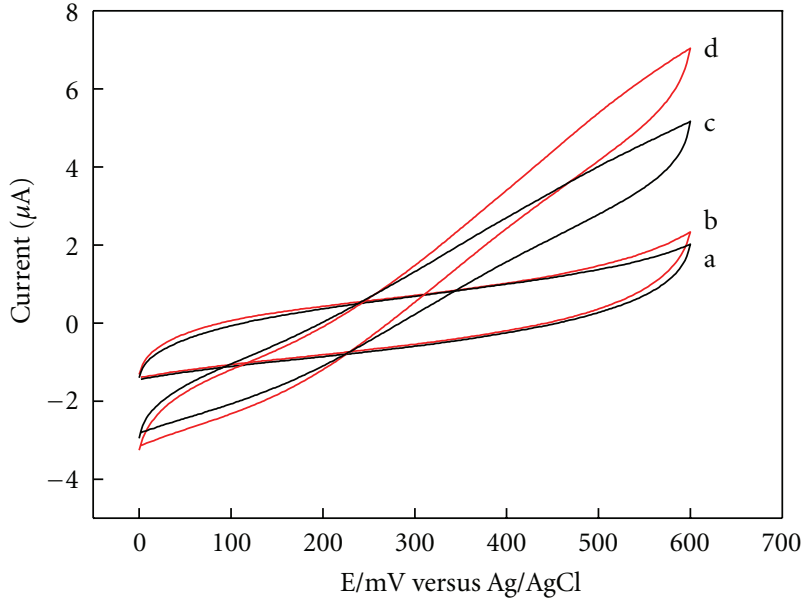

Figure 3: Cyclic voltammograms of AuNPs (a and c), MWCNTs/AuNPs ( $b$ and d) modified Pt electrode in PBS containing no choline ( $\mathrm{a}$ and $\mathrm{b}$ ), and $1.6 \mathrm{mM}$ choline $(\mathrm{c}$ and $\mathrm{d})$. The scan rate is $50 \mathrm{mV} / \mathrm{s}$. much higher than that of AuNPs/Pt electrode, indicating that MWCNTs and AuNPs in the biosensing interface efficiently improved the electron transfer between analyte and electrode surface.

Figure 4 shows a typical current-time plot of AuNPs/Pt electrode (a) and MWCNTs/AuNPs/Pt electrode (b) on successive addition of choline, which are $0.05,0.1,0.2$, $0.4,0.8$, and $1.6 \mathrm{mM}$, respectively. These amperometric data were consistent with the results obtained from cyclic voltammograms experiments, demonstrating that MWCNTs/AuNPs/Pt electrode exhibited higher activity for the enzymatic product $\mathrm{H}_{2} \mathrm{O}_{2}$ than that of AuNPs/Pt electrode. The performance of the MWCNTs/AuNPs/Pt electrode was better than that reported for MWCNTs/sol-gel modified Pt electrode and $\mathrm{ChOx} /$ sol-gel modified Pt electrode [7]. So among the resulting biosensors, MWCNTs/AuNPs/Pt electrode was the best one, which was attributed to the MWCNTAuNPs composite film. The amperometric response of MWCNTs/AuNPs/Pt electrode was in accordance with that reported for nanocomposite modified electrodes [24]. 


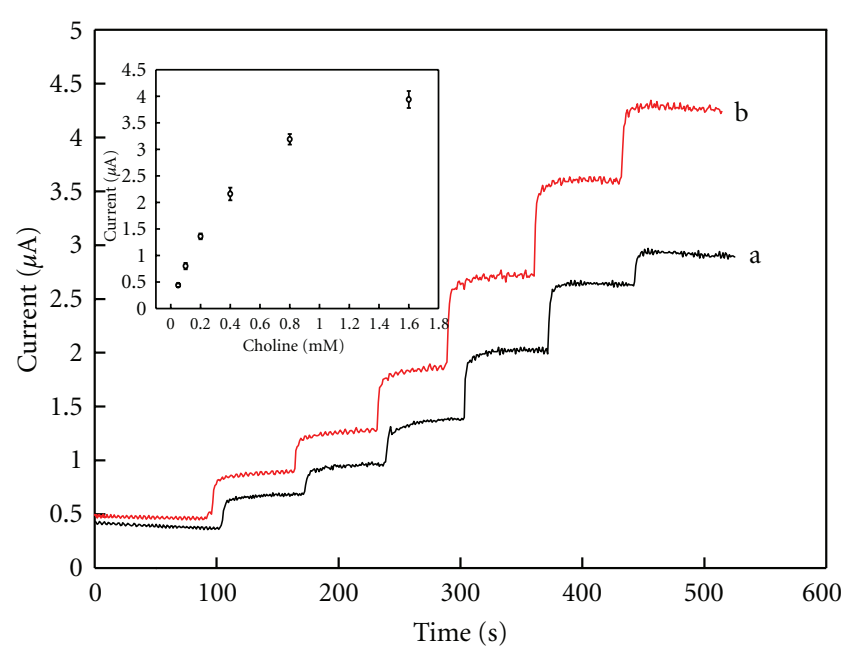

FIGURE 4: Amperometric response of AuNPs (a), MWCNTs/AuNPs(b) modified Pt electrodes upon successive addition of choline. Inset: the calibration curve of MWCNTs/AuNPs/Pt electrode. Error bars $= \pm$ standard deviation and $n=5$.

The calibration curve (Figure 4, inset) shows the current reached a saturation value at high choline concentration, showing the characteristics of the Michaelis-Menten kinetics. A linear relationship between the current and the choline concentration within the range from 0.05 to $0.8 \mathrm{mM}$ for MWCNTs/AuNPs/Pt electrode was $Y(\mu \mathrm{A})=0.49+3.56 X$ (choline, $\mathrm{mM}$ ), with a correlation coefficient of 0.9833 . The sensitivity of the electrode was $3.56 \mu \mathrm{A} / \mathrm{mM}$ with the detection limit of $15 \mu \mathrm{M}$ when signal-to-noise ratio was 3. In addition, five successive measurements of choline on MWCNTs/AuNPs/Pt electrode yielded an R.S.D. of $4.7 \%$. The stability of the MWCNTs/AuNPs/Pt electrode is evaluated by examining the current of $0.4 \mathrm{mM}$ choline. After each experiment, the electrode was washed with PBS and stored at $4^{\circ} \mathrm{C}$ when not in use. The current only decreased slightly after one month. The high stability should be attributed to the natural features of sol-gel film, MWCNTs, and AuNPs. The sol-gel film not only can prevent $\mathrm{ChOx}$ from leaking out of the electrode, but also together with MWCNTs and AuNPs can provide a favorable microenvironment for retaining the biocatalytic activity of $\mathrm{ChOx}$.

\section{Conclusions}

A novel choline biosensor based on immobilization of $\mathrm{ChOx}$ on a thiolated silica sol-gel, AuNPs, and Chit-MWCNTs interface was successfully developed. The MWCNTs/AuNPsbased Pt electrode generated a significant catalytic oxidation current compared with other $\mathrm{ChOx}$ electrodes modified with MWCNTs alone or AuNPs alone. The resulting choline biosensor showed a high sensitivity of choline, wide linear concentration range, acceptable reproducibility, and good stability. Significant advantages of the proposed choline biosensor include simplicity of fabrication and the benefits of sol-gel, MWCNTs, AuNPs, and enzyme-based amperometric biosensor.

\section{Acknowledgments}

This research is supported by the National Natural Science Foundation of China (30800261), the Scientific Research Foundation for the Returned Overseas Chinese Scholars, State Education Ministry ([2009]8), the Scientific Research Foundation of the State Human Resource Ministry for Returned Chinese Scholars (2008), and the Department of Education of Guangdong Province (Breeding Program, 2008).

\section{References}

[1] K. M. Mitchell, "Acetylcholine and choline amperometric enzyme sensors characterized in vitro and in vivo," Analytical Chemistry, vol. 76, no. 4, pp. 1098-1106, 2004.

[2] A. Guerrieri and F. Palmisano, "An acetylcholinesterase/ choline oxidase-based amperometric biosensor as a liquid chromatography detector for acetylcholine and choline determination in brain tissue homogenates," Analytical Chemistry, vol. 73, no. 13, pp. 2875-2882, 2001.

[3] S. M. Babb, Y. Ke, N. Lange, M. J. Kaufman, P. F. Renshaw, and B. M. Cohen, "Oral choline increases choline metabolites in human brain," Psychiatry Research: Neuroimaging, vol. 130, no. 1, pp. 1-9, 2004.

[4] Y. H. Bai, Y. Du, J. J. Xu, and H. Y. Chen, "Choline biosensors based on a bi-electrocatalytic property of $\mathrm{MnO}_{2}$ nanoparticles modified electrodes to $\mathrm{H}_{2} \mathrm{O}_{2}$," Electrochemistry Communications, vol. 9, no. 10, pp. 2611-2616, 2007.

[5] O. Frey, T. Holtzman, R. M. Mcnamara et al., "Enzymebased choline and l-glutamate biosensor electrodes on silicon microprobe arrays," Biosensors and Bioelectronics, vol. 26, no. 2, pp. 477-484, 2010.

[6] X. Ren, F. Tang, R. Liao, and L. Zhang, "Using gold nanorods to enhance the current response of a choline biosensor," Electrochimica Acta, vol. 54, no. 28, pp. 7248-7253, 2009.

[7] Z. Song, J. D. Huang, B. Y. Wu, H. B. Shi, J. I. Anzai, and Q. Chen, "Amperometric aqueous sol-gel biosensor for low-potential stable choline detection at multi-wall carbon nanotube modified platinum electrode," Sensors and Actuators B, vol. 115, no. 2, pp. 626-633, 2006.

[8] R. B. Rakhi, K. Sethupathi, and S. Ramaprabhu, "A glucose biosensor based on deposition of glucose oxidase onto crystalline gold nanoparticle modified carbon nanotube electrode," Journal of Physical Chemistry B, vol. 113, no. 10, pp. 3190-3194, 2009.

[9] M. Belesi, I. Panagiotopoulos, S. Pal et al., "Decoration of carbon nanotubes with $\mathrm{CoO}$ and Co nanoparticles," Journal of Nanomaterials, vol. 2011, Article ID 320516, 2011.

[10] B. Y. Wu, S. H. Hou, F. Yin et al., "Amperometric glucose biosensor based on multilayer films via layer-by-layer selfassembly of multi-wall carbon nanotubes, gold nanoparticles and glucose oxidase on the Pt electrode," Biosensors and Bioelectronics, vol. 22, no. 12, pp. 2854-2860, 2007.

[11] S. Hrapovic, Y. Liu, K. B. Male, and J. H. T. Luong, "Electrochemical biosensing platforms using platinum nanoparticles and carbon nanotubes," Analytical Chemistry, vol. 76, no. 4, pp. 1083-1088, 2004.

[12] C. Wang, G. Wang, and B. Fang, "Electrocatalytic oxidation of bilirubin at ferrocenecarboxamide modified MWCNT-gold nanocomposite electrodes," Microchimica Acta, vol. 164, no. 12, pp. 113-118, 2009. 
[13] B. Y. Wu, S. H. Hou, F. Yin et al., "Amperometric glucose biosensor based on layer-by-layer assembly of multilayer films composed of chitosan, gold nanoparticles and glucose oxidase modified Pt electrode," Biosensors and Bioelectronics, vol. 22, no. 6, pp. 838-844, 2007.

[14] V. Pavlov, Y. Xiao, and I. Willner, "Inhibition of the acetycholine esterase-stimulated growth of $\mathrm{Au}$ nanoparticles: nanotechnology-based sensing of nerve gases," Nano Letters, vol. 5, no. 4, pp. 649-653, 2005.

[15] D. N. Ventura, R. A. Stone, K. S. Chen et al., "Assembly of cross-linked multi-walled carbon nanotube mats," Carbon, vol. 48, no. 4, pp. 987-994, 2010.

[16] X. Hu, T. Wang, X. Qu, and S. Dong, "In situ synthesis and characterization of multiwalled carbon nanotube/Au nanoparticle composite materials," Journal of Physical Chemistry B, vol. 110, no. 2, pp. 853-857, 2006.

[17] H. Zhang, Z. Meng, Q. Wang, and J. Zheng, "A novel glucose biosensor based on direct electrochemistry of glucose oxidase incorporated in biomediated gold nanoparticlescarbon nanotubes composite film," Sensors and Actuators B, vol. 158, no. 1, pp. 23-27, 2011.

[18] H. D. Hill and C. A. Mirkin, "The bio-barcode assay for the detection of protein and nucleic acid targets using DTTinduced ligand exchange," Nature Protocols, vol. 1, no. 1, pp. 324-336, 2006.

[19] B. Wang, B. Li, Q. Deng, and S. Dong, "Amperometric glucose biosensor based on sol-gel organic-inorganic hybrid material," Analytical Chemistry, vol. 70, no. 15, pp. 3170-3174, 1998.

[20] J. Jia, B. Wang, A. Wu, G. Cheng, Z. Li, and S. Dong, "A method to construct a third-generation horseradish peroxidase biosensor: self-assembling gold nanoparticles to three-dimensional sol-gel network," Analytical Chemistry, vol. 74, no. 9, pp. 22172223, 2002.

[21] X. Cheng, J. Zhong, J. Meng et al., "Characterization of multiwalled carbon nanotubes dispersing in water and association with biological effects," Journal of Nanomaterials, vol. 2011, Article ID 938491, 2011.

[22] S. Bharathi, M. Nogami, and S. Ikeda, "Novel electrochemical interfaces with a tunable kinetic barrier by self-assembling organically modified silica gel and gold nanoparticles," Langmuir, vol. 17, no. 1, pp. 1-4, 2001.

[23] P. Kalimuthu and S. A. John, "Size dependent electrocatalytic activity of gold nanoparticles immobilized onto three dimensional sol-gel network," Journal of Electroanalytical Chemistry, vol. 617, no. 2, pp. 164-170, 2008.

[24] D. Du, S. Chen, D. Song, H. Li, and X. Chen, "Development of acetylcholinesterase biosensor based on CdTe quantum dots/gold nanoparticles modified chitosan microspheres interface," Biosensors and Bioelectronics, vol. 24, no. 3, pp. 475-479, 2008. 

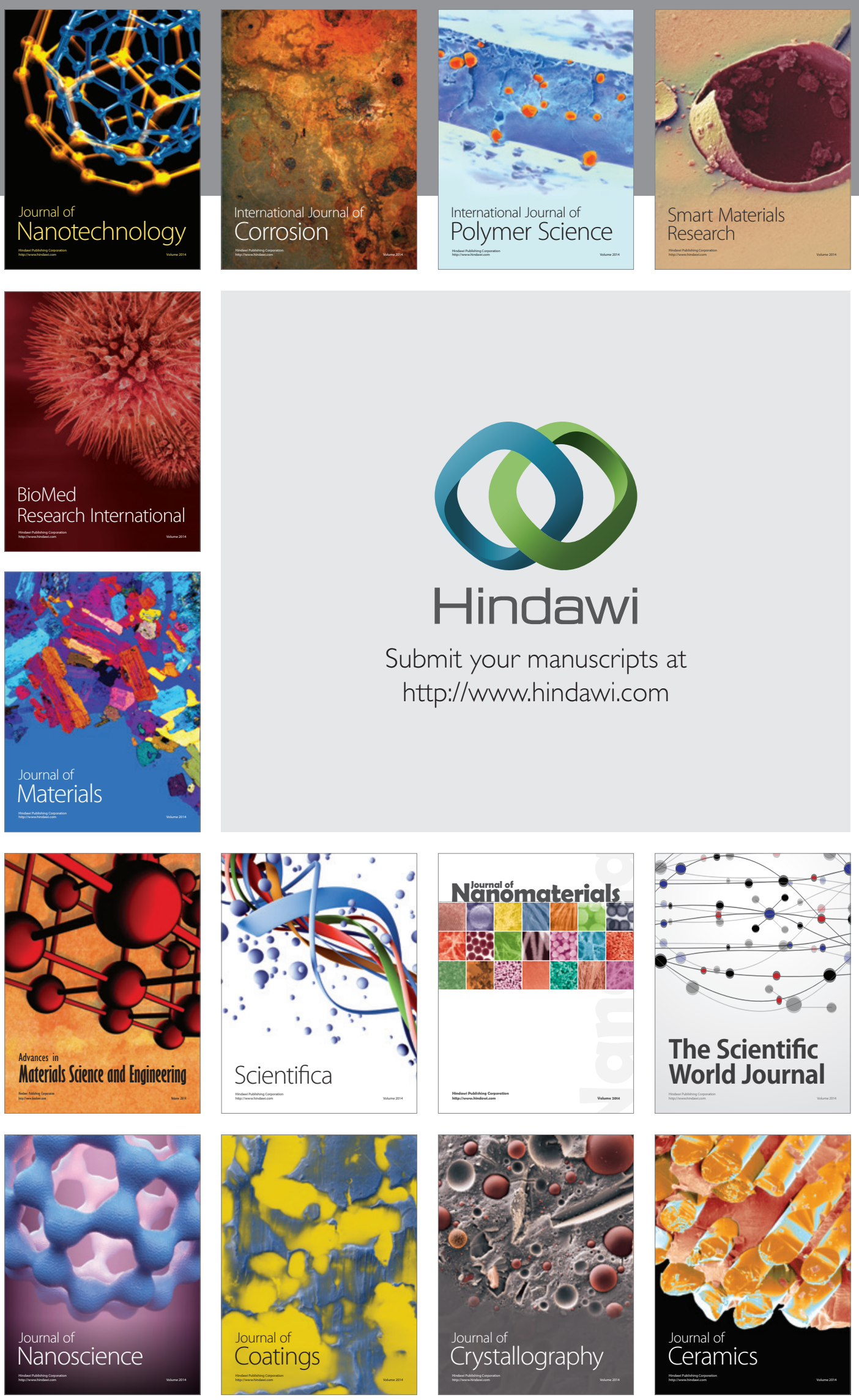

The Scientific World Journal

Submit your manuscripts at

http://www.hindawi.com

\section{World Journal}

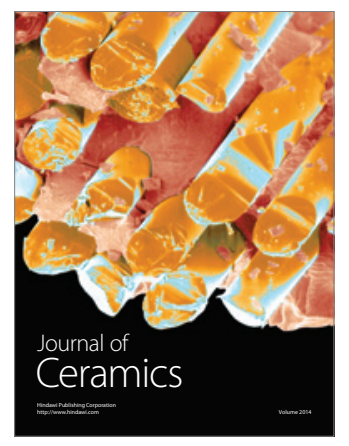

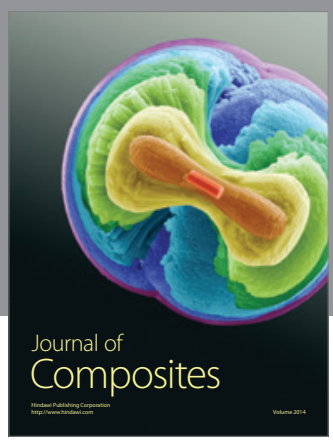
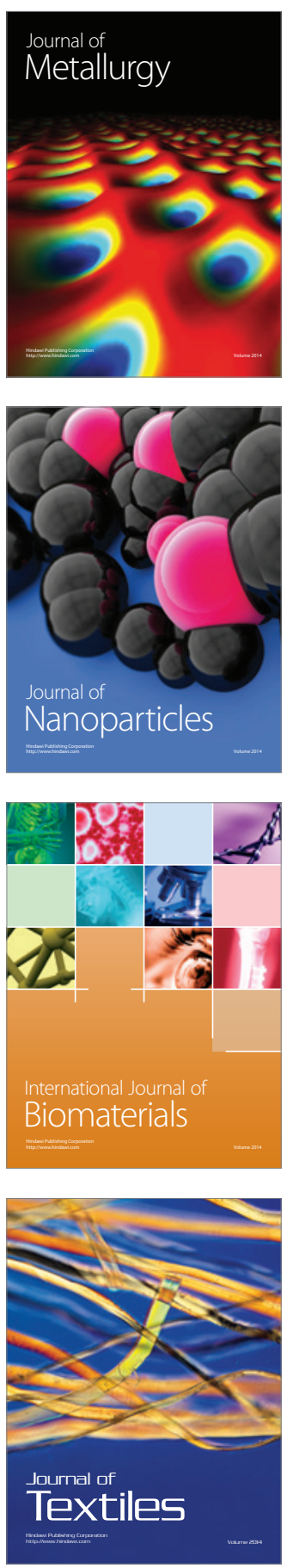